\title{
Estudio comparativo de las acciones a considerar en el proceso de diseño conceptual desde la ingeniería y el diseño de productos
}

\author{
Comparative study of the actions to consider in the process of conceptual \\ design from the engineering and the product design
}

\author{
Mauricio Guerrero Valenzuela ${ }^{1} \quad$ Bernabé Hernandis Ortuño ${ }^{2} \quad$ Begoña Agudo Vicente ${ }^{2}$ \\ Recibido 12 de noviembre de 2012, aceptado 6 de marzo de 2014 \\ Received: November 12, 2012 Accepted: March 6, 2014
}

\begin{abstract}
RESUMEN
Este artículo presenta una revisión de los modelos tradicionalmente utilizados para la realización del proceso de diseño, tanto en el diseño conceptual como en las fases que lo integran. Se comparan las concordancias y diferencias de las acciones y tareas descritas para la definición del concepto de diseño desde la perspectiva de la ingeniería frente a la de los diseñadores con el propósito de entender cómo se llevan a cabo los distintos procedimientos para su implementación. Se evidencia que las acciones que se ejecutan desde la ingeniería son precisas, descriptivas y rutinarias, en cambio desde la visión de los diseñadores los modelos revisados muestran una falta de precisión y claridad al momento de realizar las acciones y tareas conceptuales que permiten caracterizar los atributos y aspectos físicos que dan forma al producto. Se observa en el espacio del diseño que las dimensiones funcionales y morfológicas requieren de un mayor acercamiento, precisión e interacción. También se concluye que aminorar la incertidumbre en el manejo y caracterización de las ideas conceptuales es una tarea importante en la fase de diseño conceptual. Los nuevos planteamientos y modelos metodológicos deben ser descritos de una manera más clara y precisa con el objeto de optimizar y facilitar la realización del proceso de diseño, tanto en la ingeniería como en el diseño de productos.
\end{abstract}

Palabras clave: Proceso de diseño, ingeniería, modelos, diseño conceptual, producto.

\section{ABSTRACT}

This article presents a review of the traditional models used to define conceptual design both in the design process and the stages that are part of them. The concordances and differences of the actions and tasks described for the definition of the concept of design, from the perspective of the engineering as opposed to that of the designers, are compared in order to understand how the different procedures for its implementation are addressed. It is shown that the actions carried out from the engineering way of work perspective are precise, descriptive and they are addressed as a matter of routine. Nevertheless, the models reviewed show, from the designer's point of view, lack of precision and clarity in the development of the conceptual design, thus not enabling for clarification of the tasks and actions aimed to represent the characteristics and physical aspects of the concept, in order to shape the product. It is seen that the functional and morphological dimensions require a better approach, more precision and mutual interaction in the design space. It is also concluded that diminishing the uncertainty in the handling and characterization of conceptual ideas is an important task in the stage of conceptual design. The new methodological approaches and models must be described in the most clear and precise manner in order to optimize and facilitate the making of the design process both in engineering and product design.

Keywords: Design process, engineering, models, conceptual design, product.

\footnotetext{
1 Departamento de Tecnologías Generales. Facultad Tecnológica. Universidad de Santiago de Chile. C.P: 32349. Santiago, Chile. E-mail: mauricio.guerrero@usach.cl

2 Departamento de Ingeniería Gráfica. Universidad Politécnica de Valencia. C P: 46022. Valencia, España.

E-mail: bhernand@degi.upv.es; bego.agudo.vicente@gmail.com
} 


\section{INTRODUCCIÓN}

En la actualidad los modelos utilizados en el proceso de diseño abordan el análisis de sus fases desde perspectivas muy diferenciadas. Se observa falta de integración y sinergia en las acciones que definen y ordenan los procedimientos de diseño y que a su vez describen cómo se ejecutan. Al analizar gran parte de los modelos existentes suelen aparecer factores diferenciadores muy marcados como consecuencia del análisis pormenorizado de las fases y etapas que los componen, así como en lo que se refiere a la sistematización del proceso, encontrando diferencias en la manera de representar la solución final del diseño. Se observa que no abundan los modelos capaces de realizar una descripción clara del proceso de diseño, de las acciones y de los procedimientos que se utilizan para su definición en la fase conceptual, desde la ingeniería como de la perspectiva del diseñador industrial [1].

Si observamos las fases más descritas del proceso de diseño, podremos evidenciar que son aquellas relacionadas con las acciones propias de la ingeniería y las menos descritas aquellas de índole más creativa. Los procesos mentales y creativos provocan una incertidumbre patente que genera indefinición, no encontrando una formalización de procedimientos claros que nos lleven a una correcta implementación y resolución del proceso de diseño. El pensamiento creativo se asocia a instancias exploratorias y estados mentales que finalmente representan y caracterizan al producto en un espacio de interacción con el usuario, dando sentido a la solución del diseño representada por una forma, considerando que todo ello sucede en la dimensión más abstracta del proceso de diseño. El máximo discernimiento y concreción posible mediante el análisis realizado se contempla como una deseable tarea con objeto de aportar nuestra contribución en este difícil camino. El diseño conceptual se constituye como la fase asociada a un proceso mental y creativo donde se realiza la búsqueda de relaciones morfológicas que representen la solución al diseño del producto. Esta investigación de tipo cualitativo se plantea a base de una revisión bibliográfica que pretende concluir resultados respecto de la diferente visión que desde la ingeniería y desde el diseño industrial se tiene de la forma de abordar el proceso de diseño, al mismo tiempo formulando objetivos que permitan concluir un buen diseño conceptual.
La exploración de un número de modelos significativos y representativos del proceso de diseño tiene como propósito establecer la tendencia y orientación de los procesos, en la realización y resolución del diseño conceptual, así como también del espacio de diseño y de los procedimientos que comparten ingenieros y diseñadores durante la ejecución de las acciones y tareas que finalmente integran el proceso de diseño.

\section{ESTADO DEL ARTE}

Tradicionalmente el diseño conceptual se asocia a la fase de generación de alternativas mediante propuestas formales de diseño, por lo que podemos considerar esta metodología como de tipo propositiva. También podremos observar cómo aparecen claramente identificados aquellos modelos con pensamiento funcional de tipo reduccionista, enfocados a la temporalidad del proceso, y que intentan dar respuestas rápidas al diseño según las necesidades del usuario. Por lo general estos partirán de parámetros funcionales y fijarán objetivos a cumplir. La realización típica secuencial de las fases del proceso, como lo muestra la Figura 1, se realiza a partir de las especificaciones del producto (EDP), las que establecen los requerimientos y parámetros para definir el concepto de diseño.

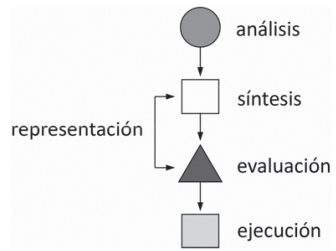

Figura 1. Diagrama del proceso de diseño secuencial, adaptado de Rivka [2].

También se han escrutado modelos que consideran los atributos técnicos y que justifican la búsqueda de la solución del objeto tridimensional desde la etapa proyectual. Estos operan mediante el cribado de ideas conceptuales, que se manifiestan en una representación estético-formal (morfología) a partir de volúmenes preliminares definidos al inicio del proceso (diseño básico). Algunos modelos hacen énfasis en generar lo antes posible soluciones de diseño [3], se suelen centrar desde el inicio del proceso en la solución y posteriormente en el análisis de esta solución. Suelen ser modelos sencillos enfocados a la reducción de fases contemplando las 
actividades que deben realizarse durante el proceso de diseño, son los llamados modelos descriptivos. Estos modelos permiten identificar las fases del proceso de diseño tradicionalmente aceptadas, como se observa en la Figura 2. Los modelos descriptivos intervienen en el proceso por medio del diseñador de manera simple y secuencial, como lo haría el método científico de prueba y error, o de ensayos heurísticos en busca de la solución.

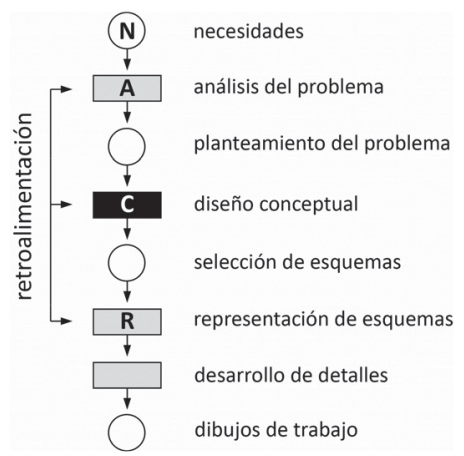

Figura 2. Modelo descriptivo del proceso de diseño, adaptado de French [4].

$\mathrm{Al}$ observar estos modelos veremos que permiten comprender el problema de diseño y ordenar la información generada en el proceso mediante el análisis del espacio de diseño. Usualmente no permiten identificar un plan de acción para su ejecución. Existen también modelos centrados en el diseño de conjunto, estos dan pautas de cómo desarrollar las fases de implementación, y son denominados modelos prescriptivos, incorporan en las fases y acciones del proceso de diseño los procedimientos, tareas, y actividades necesarias para un buen análisis, desarrollo y ejecución del mismo. También existen modelos que denominaremos como orientados a la creatividad, los que permiten considerar de manera simple e intuitiva los procedimientos en las acciones conceptuales inherentes del diseñador al momento de diseñar.

El diseño se plantea como una actividad sistemática que permite identificar las necesidades del cliente y el mercado, controlando los procesos que consigue desarrollar el producto [5]. En esta tipología podemos encontrar los modelos denominados axiomáticos [6], cuya orientación sistemática demuestra que responde a bases científicas para la consecución del diseño, sintetizando todas las actividades del proceso y los requisitos funcionales en los denominados dominios técnico-funcionales, dejando al dominio físico todas aquellas tareas y acciones que complementen la solución desde la ingeniería. Su base fundamentalmente teórica deja claro que el propósito es vincular la forma con la función.

Igualmente los modelos prescriptivos [7] proponen integrar las especificaciones y atributos que conducen eficientemente a la fabricación del producto. Proponen un input desde las necesidades del cliente y a continuación el desarrollo de la fase de diseño conceptual, generando en esta etapa el concepto de diseño. Estos modelos no dan solución a los subproblemas que pudieran generarse con el diseño, sobre todo donde adquieren importancia los aspectos morfológicos y la representación de los subsistemas físicos, los que deben interactuar con los sistemas funcionales. Los factores que no vienen delimitados por la ingeniería dificultan su integración en la fase de diseño conceptual, principalmente debido a que es la parte más abierta y abstracta del proceso de diseño.

\section{Proceso de diseño conceptual: definición del concepto}

La palabra diseño, según Alcaide y Artacho, está limitado a la forma y su representación externa [8] (color, textura, etc.), pero no al artefacto en su conjunto. En cambio design, para el anglosajón, hace referencia a todas las actividades y acciones para el desarrollo de una idea de producto (cumplir un propósito), entendiéndose más como un proyecto: el diseño constituye un proceso complejo de búsqueda de alternativas y definición material de las mismas [9]. El significado terminológico en la etapa proyectual, según quién diseña, ingenieros o diseñadores, será lo que determine la solución final: para el diseñador concepto del diseño (producto), para el ingeniero concepto de diseño (propósito o meta). Parece ser que definen lo mismo, pero la interpretación de las tareas y acciones del proceso dependiendo de quién las ejecuta son diferentes. Ir desde la idea inicial hasta el producto hace que los modelos utilizados determinen y representen la solución forzadamente. También debemos constatar que los procesos dependientes del azar no van ligados con lo racional y viceversa. 
Se considera que economía y máxima eficiencia es la respuesta al diseño desde la ingeniería, mientras que diseño industrial, según Farr (citado por Tovey [10]), considera la implicación de las personas, por lo que el diseño industrial se plantea como un proceso probablemente más correcto al identificarlo desde el área de la responsabilidad y expectativas emocionales del usuario.

En el desarrollo de productos [11] las especificaciones bien definidas permiten usualmente generar un buen concepto de diseño, especialmente donde la definición de volúmenes preliminares y estructuras son determinantes a la hora de dar forma al producto. El proceso de diseño según diversos autores es creativo e impredecible en la fase conceptual [12], y por tanto la obtención de una buena respuesta de diseño también lo es. La exploración del espacio conceptual comúnmente es de tipo divergente, por lo que se producen un número mayor de alternativas e ideas, permitiendo llegar a mejores y nuevas respuestas para dar con la solución al diseño. Se puede establecer, por tanto, que las relaciones existentes en la mayoría de los modelos actualmente utilizados para la definición del diseño conceptual resuelven eficientemente los propósitos del problema de diseño al amparo de la ingeniería, dando importancia al diseño denominado como creativo y a sus acciones solo cuando se activan o se hacen necesarias aquellas tareas que posteriormente determinan la apariencia y la forma del producto. La culminación de la respuesta de diseño es una descripción del artefacto que se va a fabricar [3]. Este enfoque indicaría que es desde la ingeniería donde se sientan las bases para definir y diseñar un producto. Por otra parte, indica Cross (2002), el diseño industrial y el diseñador no importa cómo trabajen, siempre y cuando produzcan la descripción final del producto diseñado. El propósito del diseño es dar la forma física al producto [11], proporcionándole las tareas de preocuparse de la estética, la ergonomía y de las interfaces relacionadas directamente con el usuario. Una gran cantidad de ideas lleva a un mayor éxito en la solución obtenida (Ehrlenspiel, Dylla, 1993, citado por Mulet [12]). Se entiende entonces que desde el punto de vista de la ingeniería, la amplitud del espacio de diseño[19] optimiza la definición del concepto, permitiendo encontrar nuevas ideas que aumentan las probabilidades de éxito, no para generar nuevos fines para el diseño, ya que estos vienen definidos teóricamente por las especificaciones y funciones. Dym y Little [7] afirman al respecto que la ampliación útil del espacio de diseño es necesario para cumplir con las metas solicitadas y satisfacer solo el propósito.

El método de cinco pasos de Ulrich [11] para el desarrollo del concepto: clarificar el problema; búsqueda externa; búsqueda interna; exploración y reflexionar soluciones. O el método de cuatro pasos de Otto [13]: necesidades; sistemas de funciones; soluciones y construcción de las soluciones, proponen estados intermedios de acciones para determinar el concepto de diseño. En la Figura 3 se observa un modelo típico de las tareas que generalmente se desarrollan en este tipo de métodos para la definición del concepto de diseño.

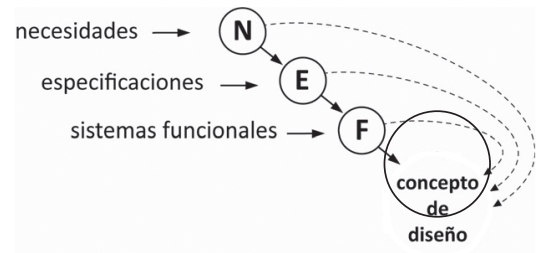

Figura 3. Modelo idealizado para la definición del concepto de diseño. Elaboración propia.

Según lo expuesto se puede determinar que el proceso de diseño conceptual no responde a una única orientación para la definición del concepto, sino que depende del enfoque inicial, de cómo se enfrenta el proceso según la estrategia de diseño y de quién lo realiza:

a) Hacia el diseño mecánico, que se define a partir de aspectos técnicos, funcionales y mecánicos, de software, eléctricos, etc., que cumplen con el propósito de la ingeniería.

b) Hacia el desarrollo de productos, cuando se desarrollan todas las fases del proyecto industrial (empresa), incluye la planeación del producto, la ingeniería del diseño, la gestión entre otras.

c) Hacia el diseño formal, cuando se orienta hacia la morfología, el estilo, la estética y apariencia del producto, centrándose en el usuario, el uso e identidad que se percibe de un producto. 


\section{EL PREDOMINIO FUNCIONAL EN EL PROCESO DE DISEÑO CONCEPTUAL}

Se considera que la creación proyectual es un proceso de algo, para llegar a una cosa. Para que esto ocurra, y ese algo exista se debe saber qué hacer y cómo hacerlo, por lo tanto satisfacer la necesidad preliminar de establecer un orden en generar una estructura para cumplir con ese objetivo es fundamental. Nos enfrentamos a un problema que debe ser analizado y descrito con objeto de obtener una única solución coherente que cumpla con todos los requerimientos y parámetros definidos previamente.

La creación comienza con las siguientes premisas: determinar el problema y solucionar el problema. Para ello se recurre a dos procesos fundamentales para el diseño y la creación: el análisis y la síntesis. Cada uno de ellos tiene sus propias acciones y tareas, las que se realizan mediante algún método y cuyo resultado permite representar una realidad que antes no existía. Si bien existe una mayor recurrencia hacia la utilización de modelos basados en métodos científicos para la resolución de problemas, no se debe dejar de lado el método creativo. Desde esta perspectiva es interesante entender y visualizar cuáles son los factores que intervienen en la fase creativa, las acciones que se realizan en el dominio funcional, y cómo se generan los conceptos a partir de la descripción racional de un problema.

El espacio de diseño conceptual requiere de procesos bien definidos y el lenguaje contextual y visual debe ser capaz de representar morfológicamente la solución de diseño. La claridad semántica y lingüística en la terminología utilizada debería ser compartida por ingenieros y diseñadores, y debiera ser expresada y representada por formas análogas, y por tanto comunes, con objeto de optimizar la solución final. El diseño conceptual se inicia con la sinergia entre las especificaciones y los objetivos que se circunscriben al producto [14], con los requerimientos técnicos, restricciones y variables del diseño tal y como se observa en la Figura 4. El modelo conceptual determinado por la ingeniería no tendrá forma física ni transformará las expectativas de las ideas en una representación formal hasta que no suceda lo siguiente:

a) Hasta que el proceso de diseño esté finalizado. b) Hasta que la forma final del producto sea definida por el diseñador.

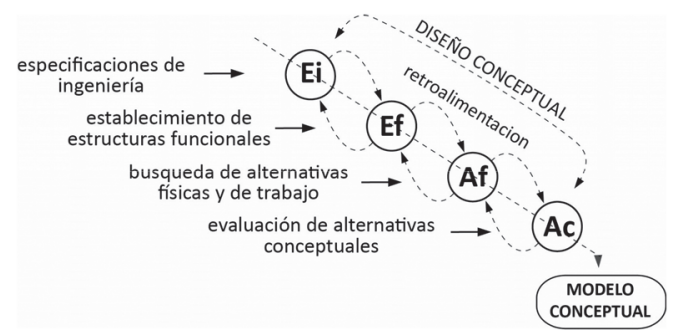

Figura 4. Etapas del diseño conceptual, adaptado de Chaur B., Tesis Doctoral, UPC [14].

El proceso de diseño comienza con un propósito y concluye con la obtención del resultado de ese propósito. Para ello se ha especificado y documentado toda la información necesaria para su fabricación (Asimow, citado por Mulet) [12]. El diseño en la ingeniería [15] es un proceso sistemático, inteligente en el cual los diseñadores generan, evalúan y especifican los conceptos para los dispositivos, los sistemas, o los procesos, cuya forma y función alcanzan los objetivos de los clientes o las necesidades de usuarios mientras satisfaga al sistema especificado. La eficiente identificación de las necesidades del cliente [11] está estrechamente asociada con el establecimiento de las especificaciones, la selección y generación del concepto.

El modelado funcional del proceso [13] da paso a la transformación de las necesidades en especificaciones para el diseño. Las acciones iniciales permiten la descripción precisa de los objetivos del producto en la fase preliminar de análisis. Ulrich [11] las llama especificaciones objetivo, constituyéndose en una de las directrices que definen el propósito del diseño: Las especificaciones definidas en la fase inicial del proceso establecen el propósito del producto. Las funciones se interpretan como el sustento del concepto de diseño permitiendo el flujo de información y la generación de la base funcional en la estructura del producto. En definitiva implica que el dispositivo o artefacto haga lo que debe hacer atendiendo a la finalidad para la que se ha concebido. La función de un producto, señala Otto y Wood [13], es la relación reproductiva entre la entrada disponible y la salida deseada de un producto (la solución), independiente de la forma particular final que se le otorgue. 
Al determinar el problema de diseño se deben establecer los modelados funcionales [16], el problema se divide en subproblemas, lo que facilitaría el entendimiento y los procedimientos que se realizarán durante el proceso, entonces debe ocurrir lo mismo con las funciones, se subdividen en funciones secundarias, lo que facilitaría la búsqueda de la solución haciendo menos complicada su resolución. Por lo general los modelos consideran de manera diferente el análisis inicial para la identificación y establecimiento de las funciones. En el modelo de Pugh [5], las funciones están en la fase de especificaciones y formulación, en otros [17], en la fase de desarrollo de las especificaciones del diseño. Para Cross [3] las funciones se sitúan en la fase de exploración y generación. En el modelo de evolución funcional (FBS), propuesto por Gero [18] que se observa en la Figura 5, la función, el comportamiento y la estructura se consideran subespacios o abstracciones del proceso cuyos objetivos se transformarían en una descripción del diseño. La función describe la finalidad del diseño, el comportamiento describe el funcionamiento y la estructura describe la solución (citado por Mulet) [12].

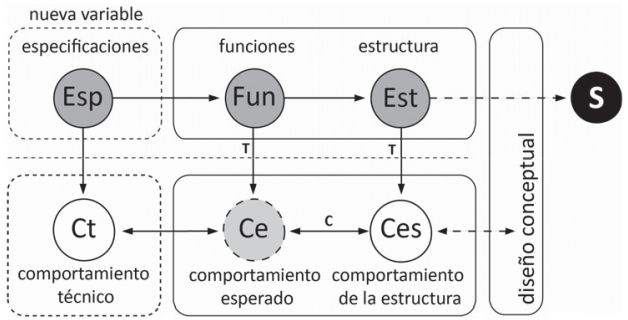

Figura 5. Modelo de evolución del proceso de diseño a partir de funciones, adaptado de Gero [18].

Se observa que no hay grandes diferencias en la manera de abordar las tareas en el proceso a partir de las funciones y especificaciones. Estas dicen lo que se debe hacer y en qué momento de la fase se debe trabajar. Los subproblemas y los procedimientos aparecen en el proceso y presentan las acciones para el logro. Los métodos $\mathrm{y}$ herramientas existentes son muchos y variados, $\mathrm{y}$ la decisión de cuál utilizar depende del enfoque, de la estrategia y de la envergadura del proyecto. Las restricciones impuestas, normativas, tecnologías, costos, etc., permiten la definición del producto y su desarrollo posterior llegando hasta la etapa de fabricación del producto.
El enfoque metodológico de tipo sistémico (B. Hernandis [19]) responde de manera óptima a la definición de las limitaciones en las acciones y tareas necesarias para la resolución del diseño. La ventaja de esta metodología es su flexibilidad en el momento de trabajar en el espacio de diseño, ya que contempla al mismo tiempo todos los subsistemas que se relacionan con el procedimiento.

El modelo sistémico, como se observa en la Figura 6, integra las acciones en las dimensiones fundamentales del diseño: forma, función y ergonomía. Realiza de manera global el desarrollo del producto, y engloba tanto a la fase de diseño conceptual como a la de detalle. El modelo estimula el pensamiento creativo en tres fases: pensamiento convencional, división simultánea e integración, permitiendo visualizar, en todo momento, los aspectos del espacio del diseño e interactuar rápidamente en el tratamiento y ordenamiento de la información según lo requieran los distintos subsistemas propuestos por el modelo.

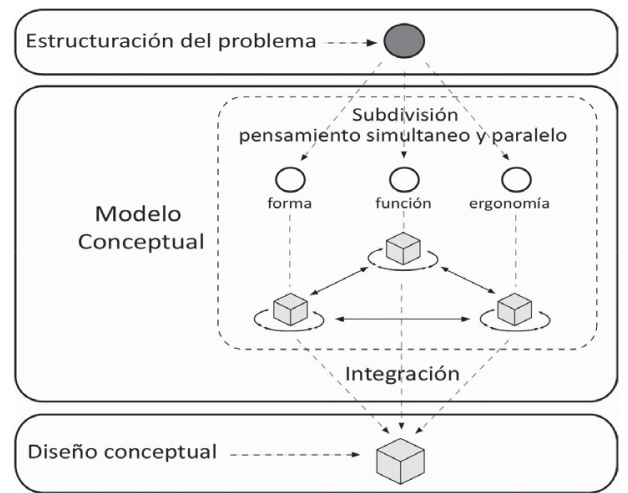

Figura 6. Modelo de B. Hernandis y J.C. Briede [19].

Distinta es la propuesta de Ulrich y Eppinger [11], en ella el proceso genera una gran cantidad de información y debe ser sistematizada de manera lógica y racional. Este enfoque secuencial, como lo muestra la Tabla 1, permite determinar la información necesaria de entrada y salida de datos en el proceso.

Asociar las funciones con especificaciones permite determinar los objetivos del proceso: generar las directrices, las acciones y tareas para el desarrollo del concepto de diseño. Las funciones son factores clave para dar la partida a la fase de diseño conceptual, y deben estar en concordancia con la definición previa de los 
Tabla 1. Información y proceso de diseño, Adaptado de Wodehouse [20].

\begin{tabular}{|l|l|l|}
\hline \multicolumn{1}{|c|}{ Fase } & \multicolumn{1}{|c|}{$\begin{array}{c}\text { Ejemplos de la } \\
\text { información } \\
\text { de entrada }\end{array}$} & \multicolumn{1}{c|}{$\begin{array}{c}\text { Ejemplos de la } \\
\text { información } \\
\text { de salida }\end{array}$} \\
\hline Planificación & $\begin{array}{l}\text { Datos del mercado, } \\
\text { reportes de la } \\
\text { empresa }\end{array}$ & $\begin{array}{l}\text { Informes, documentación, } \\
\text { planificación, comunicación } \\
\text { general del plan }\end{array}$ \\
\hline $\begin{array}{l}\text { Desarrollo } \\
\text { del concepto }\end{array}$ & $\begin{array}{l}\text { Competencia del } \\
\text { mercado, diseño de } \\
\text { esquemas }\end{array}$ & $\begin{array}{l}\text { Técnicas, bocetos, dibujos, notas de } \\
\text { reuniones de equipo, comunicación }\end{array}$ \\
\hline $\begin{array}{l}\text { Diseño a nivel } \\
\text { de sistema }\end{array}$ & $\begin{array}{l}\text { Patentes diseño } \\
\text { previo de esquemas }\end{array}$ & $\begin{array}{l}\text { Bocetos, dibujos, costos, maquetas, } \\
\text { modelos físicos }\end{array}$ \\
\hline $\begin{array}{l}\text { Detalles de } \\
\text { diseño }\end{array}$ & $\begin{array}{l}\text { Catálogos, planos } \\
\text { de fabricación, } \\
\text { diagramas de comp. }\end{array}$ & $\begin{array}{l}\text { Dibujo de detalles, cálculos, } \\
\text { sólidos, modelos matemáticos, } \\
\text { modelos 3D }\end{array}$ \\
\hline $\begin{array}{l}\text { Prueba y } \\
\text { refinamiento }\end{array}$ & $\begin{array}{l}\text { Estandarización, } \\
\text { base de datos }\end{array}$ & $\begin{array}{l}\text { Datos experimentales, manufactura, } \\
\text { materiales, especificaciones, } \\
\text { ensamble }\end{array}$ \\
\hline Producción & $\begin{array}{l}\text { Consumidor, } \\
\text { retroalimentación, } \\
\text { datos de venta }\end{array}$ & $\begin{array}{l}\text { Presentaciones de venta, } \\
\text { instrucciones, fotos, presentaciones } \\
\text { gráficas }\end{array}$ \\
\hline
\end{tabular}

requisitos y las necesidades, tanto del usuario como en la planificación del proyecto.

\section{PENSAMIENTO Y PROCESO CREATIVO EN EL DISEÑO CONCEPTUAL}

La creatividad implica por lo general hacer algo que es nuevo [21] e incorpora en la solución de diseño algo del dominio y la experiencia que está en la mente del ser creativo. La creatividad se manifiesta en la ingeniería, las artes y las ciencias; dependerá del enfoque y objetivo que se tenga, así como del área y espacio donde se despliegue para que el proceso creativo dé como resultado muchas alternativas para la solución de diseño. De Bono [22] comenta que algunos consideran que la creatividad es tener talento, $\mathrm{y}$ que el pensamiento creativo tiene que ser raro, pero esto no tiene nada que ver con la naturaleza de la creatividad. Uno de los objetivos fundamentales del pensamiento creativo es poder descubrir nuevas y mejores maneras de hacer las cosas. Los modos de diseñar según De Bono son dos, el modo habitual, que plantea los requisitos y con ellos se puede generar el molde para luego tratar de encontrar algo que se amolde a ellos, lo que permite que la creatividad del diseñador sea dirigida a soluciones conocidas; y el modo conceptual, que permite desarrollar conceptos creativos, y luego ver cómo se les da forma satisfaciendo los requerimientos de entrada. En este caso las especificaciones modelan el concepto, y si el pensamiento creativo se orienta al diseño aumentan las expectativas y la cantidad de posibles soluciones para dar respuesta a algo que no existe.
La creatividad parece ser de naturaleza subjetiva, ilógica y azarosa, pero no lo es. Cualquiera puede sumergirse en la creatividad libremente, pero las técnicas sistematizadas y organizadas mentalmente formalizan la creatividad. La creatividad es orden mental y de pensamiento, requiere dirección y sentido, si no se tiene un orden, un conocimiento y un comportamiento lógico y deliberado de la causa que lo origina (la motivación), difícilmente podrá generar pautas y patrones creativos que permitan al diseñador dar respuestas creíbles y confiables. La creencia [23] de que cuanto menos condicionantes y requisitos se tengan se es más creativo, es errada, la creatividad debe abrir espacios de búsqueda, es decir, mientras más condicionantes existan inicialmente (a partir del análisis del problema), mayor será la capacidad creativa para proponer alternativas y dar solución al diseño. En cambio, para los sistemas observados desde la ingeniería la creatividad es considerada de naturaleza resolutiva técnicamente, la funcionalidad de los conceptos debe ser bosquejada como respuesta a las especificaciones que representan el problema y el propósito del diseño, determinación del problema de diseño. Por lo general esta fase se representa mediante esquemas, dispositivos o sistemas de configuraciones funcionales y técnicas. La creatividad [24] es un aspecto de fascinación y estimulación del ser humano y de su pensamiento, $\mathrm{y}$ tiene la capacidad de reestructurar viejas ideas y aplicarlas a una nueva.

El pensamiento creativo alberga en sí mismo los procesos cognoscitivos relacionados con las soluciones. Se asocia a la exploración y percepción que inicialmente describe el análisis del problema y de la idea que se tenga para que pueda ser representada mediante una imagen preliminar en la mente. Con esto se instalan las condiciones y estructuras morfológicas del objeto para caracterizarlo físicamente en el espacio de interacción objetousuario (espacio del diseño).

Estas relaciones y asociaciones mentales pueden ser convencionales o no convencionales, y dependiendo del ámbito y contexto del problema será resuelta su expresión formal. Por otra parte, de los espacios conceptuales emergen abstracciones, imágenes y objetos permanentemente, lo que permite al diseñador introducirse en la fase de diseño generativo gracias a la experiencia y entrenamiento previo que este haya tenido. La solución generada se da en la acción creativa como se observa en la Figura 7. 


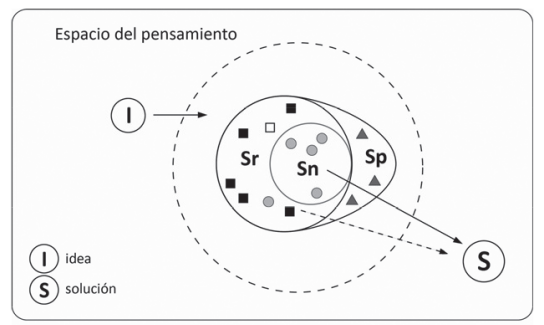

- Soluciones realizadas $(\mathrm{Sr})$ : experiencias guardadas

- Soluciones nuevas $(\mathrm{Sn})$ : experiencias nuevas

- Soluciones prescindibles $(\mathrm{Sp})$ : experiencias intrascendentes

Figura 7. Modelo teórico del pensamiento y acción creativa. Adaptado de Eckert [25].

Las soluciones conceptuales que se adopten como definitivas deben haber pasado por un proceso mental estructurado desde las operaciones [26] que se realizan: dirección, evaluación y decisión para proponer la solución que permitirá el mejor resultado y camino posible, pudiendo encontrarse en cada uno de los pasos las secuencias y acciones del proceso creativo, independientemente del modelo utilizado.

La naturaleza repetitiva de los modelos creativos permite complementar o redefinir un concepto de diseño y su solución, mejorando los resultados en el transcurso del proceso. El pensamiento creativo aporta las pautas para un comportamiento creativo en el diseño. Si el creador compromete sus sentidos y toda su actividad en el proceso que lleva adelante, lo más probable es que sus acciones y resultados sean adecuados. Si no es así, reflejará en su proceso creativo la no utilización de factores cognoscitivos para la resolución de un modelo conceptual. Por lo tanto podemos decir que la creatividad es un proceso mental en el espacio del pensamiento (pensamiento creativo) y que al mismo tiempo se observa en el objeto representado en la forma de la solución final (representación de la forma).

\section{El proceso creativo en el diseño}

El alcance del estudio no propone adentrarse en los laberintos de la creatividad, del pensamiento creativo o de los procesos cognoscitivos del ser humano, sino que pretende entender la creatividad desde la perspectiva de su aplicación en los procesos del diseño conceptual y de las acciones comúnmente utilizadas durante dicho proceso. De Bono [22] dice que el proceso creativo normal es como un camino principal continuo, la creatividad es encontrar, o que aparezcan, caminos laterales (input y output) que permitan extender las posibilidades de crear. Por su parte, Hernandis y Briede [19] comentan que cada proceso innovador es acompañado por un proceso creativo y que las soluciones se pueden encontrar en cualquier momento del proceso. El pensamiento cíclico, como se observa en la Figura 8, responde a esta premisa, y los resultados obtenidos demuestran que un modelo sistémico logra integrar mejor el objetivo de diseño señalado desde el inicio del proceso, permitiendo iterar dinámicamente en la búsqueda de la mejor respuesta de diseño, y retroalimentar las alternativas propuestas por el diseñador.

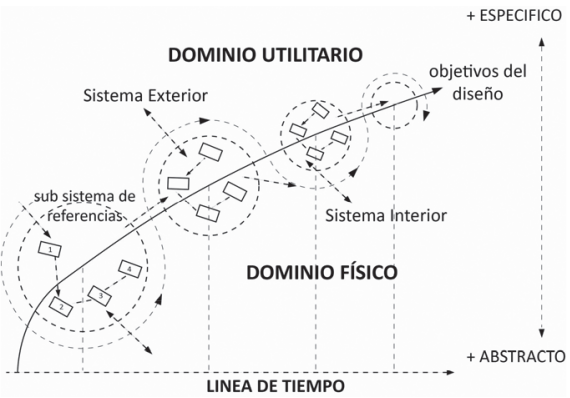

Figura 8. Modelo del pensamiento cíclico, Hernandis y Briede [19].

Las acciones proyectuales y exploratorias convergen y divergen a lo largo de la trayectoria del proceso. La iteración permanente de los resultados que se van obteniendo, y la división de problemas en subproblemas y en otras acciones determina las características más significativas del proceso creativo. Para el pensamiento creativo [27] existen dos tareas explícitas: la determinación del problema y la solución del problema: Problem Setting y Problem Solving, ambas están relacionados con actividades mentales: la racionalidad y el conocimiento. El espacio de búsqueda mental por lo general se asocia a las soluciones esquemáticas del proceso proyectual, y deja de lado en la resolución del problema los aspectos y cualidades formales que atañen a las personas. El vínculo entre el pensamiento creativo y el pensamiento lógico, a partir de un hecho teórico científico, frente a un hecho interpretativo emocional, intenta establecer el diálogo entre lo racional y lo irracional haciendo patente que todo proceso proyectual pasa por una actividad mental y creativa. El reto consiste por tanto en dar forma y sentido a las expresiones físicas, cualidades y características de cada nuevo objeto proyectado, considerando que este, a su vez, dependerá de factores como materiales, nuevos procesos de fabricación y nuevas tecnologías entre otros a considerar. 
La innovación generada debe integrar las variaciones en el sistema producto como resultado del proceso realizado, lo que se traduce en nuevas formas y aspectos físicos que se integren con las estructuras funcionales y técnicas en la solución de diseño. El miedo a lo desconocido y la falta de credibilidad interna de las propias capacidades creativas llevan muchas veces a definir y dar forma a un producto mediante parámetros funcionales y técnicos validados por la ingeniería y garantizados por el rigor de los datos y especificaciones que se definen en el proceso de diseño.

Se asume que las dimensiones del diseño conceptual y el espacio en donde se desenvuelve son consideradas por quien diseña como propias a su área de intervención. En estas circunstancias la determinación de un producto y del proceso no es coincidente, muchas veces carecen de importancia aquellas acciones que reducen los tiempos para dar la solución al diseño. La secuencia de tareas en el proceso de diseño se ve dificultada por la búsqueda de representaciones adecuadas que interpreten aquellos rasgos formales que, unidos básicamente a la función, pueden definir eficientemente la solución formal del diseño.

\section{Modelos para el concepto}

El modelo de síntesis conceptual propuesto por Plsek [28] es el que mejor representa el proceso creativo, como lo muestra la Figura 9. Los dominios y espacios conceptuales están identificados en un proceso cíclico de cuatro pasos, representando y describiendo las acciones y subacciones que permiten generar las ideas y las soluciones. Identifica las tareas que se deben realizar secuencialmente, pero no dice cómo actuar con ellas en los procedimientos y acciones para la correcta interacción entre las diferentes fases.

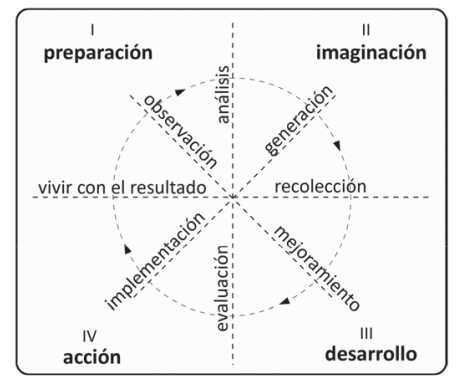

Figura 9. Ciclo de síntesis del proceso creativo, Plsek [28].
El propósito del diseño preliminar [29] es determinar qué alternativas son mejor valoradas para determinar el concepto de diseño. Por su parte, Wood [13], en su modelo, le da importancia a la generación del concepto y a toda la información relacionada con el análisis formal. Sugiere un proceso mental de repetición desde el análisis morfológico, lo que permitiría incluir conceptos adicionales y secundarios para la generación del concepto de diseño. Pugh [5] dice que la fase conceptual del diseño tiene dos ciclos: generación y evaluación de la solución. Este modelo simplista deja muy abierto el camino mental y carece de rigurosidad al momento de su aplicación. El modelo "C-K" [30] propone que la resolución del diseño se aborde desde dos metadimensiones de distinta naturaleza: la analítica (espacios de alternativas del concepto, C), y la representativa (espacio del conocimiento, K). El resultado presenta diseños más rigurosos y abarca cada uno de los factores en ambos espacios. Es un modelo que se identifica entre función y espacio físico no lineal, y que como la gran mayoría de los modelos que interactúan dinámicamente se alimenta con cada una de las nuevas alternativas que se generan.

La revisión bibliográfica realizada se ha basado en los modelos más representativos y tradicionalmente utilizados. Estos incorporan en sus fases de desarrollo consideraciones de tipo análogas al momento de entrar en la borrosa fase creativa y conceptual. La poca rigurosidad con que se aborda el proceso creativo hace pensar que no se cuenta con las herramientas adecuadas que faciliten el entendimiento multidisciplinario, y que habitualmente los requisitos y atributos que contienen las especificaciones no pueden ser plasmadas en un objeto tridimensional que cumpla con los requerimientos y expectativas iniciales. Los principios del diseño creativo y los patrones de comportamiento tanto en la ingeniería como en el diseño deben ser colaborativos e integrados para facilitar el proceso y la exploración del espacio de diseño. La abstracción y el manejo de las ideas deben dar resultados novedosos, además deben proporcionar una iteración permanente entre los dominios funcionales y físicos, donde la racionalidad comparta su espacio con la subjetividad controlada de los procesos mentales integrándose finalmente con el de los objetos y sus representaciones físicas. 
La oferta de métodos, técnicas y herramientas para el diseño son eminentemente lógicos y racionales y su fin último es cumplir con los requisitos, validar las respuestas y las acciones que se desprenden del propio proceso. En muchos de ellos el establecimiento de las fases se centran principalmente en analizar el problema; sintetizar y evaluar las respuestas $l o$ que debemos hacer, sin embargo cuando queremos saber cómo hacerlo el escenario es borroso. Los dominios menos claros son aquellos en donde gobiernan las ideas, abstracciones y los procesos mentales, pero principalmente en la definición conceptual, en donde se representa el concepto de diseño y el objeto. La opinión generalizada es que el espacio conceptual no es un campo que puede ser definido claramente. El límite y proximidad que tienen la forma y la función desde un punto de vista conceptual evidentemente dificulta su trazado.

\section{METODOLOGÍA}

Se ha optado por realizar una revisión bibliográfica sobre la literatura fundamental que analiza las diferentes perspectivas del diseño conceptual, desde la orientación de la ingeniería y desde la que señalan los diseñadores. Para ello se ha analizado y comparado un grupo representativo de los principales modelos que existen, como lo muestra la Tabla 2. Con el objeto de establecer las concordancias y diferencias principales se han codificado estos modelos a fin de facilitar la notación en la investigación y sus resultados. Según los modelos analizados, si observamos y comparamos desde lo cualitativo lo consignado en la Figura 10, se puede concluir que las fases relacionadas con el diseño conceptual son representadas por una zona continua difusa que no deja claro cuáles son las acciones y tareas que deben realizarse y mucho menos cuándo se les da inicio y término. Por el contrario, en el momento de abordar las tareas iniciales y finales del proceso no se encuentran grandes dificultades para su realización, fundamentalmente porque son tareas rutinarias y típicas de procesos de ingeniería, las cuales están descritas en la mayoría de los modelos, lo que sugiere considerar que estas acciones y tareas efectivamente tienen procedimientos claros y precisos a la hora de realizarlas, pudiendo repetirse tantas veces sea necesario.

Las tareas y acciones en la dimensión funcional son rutinarias y se van realizando muchas veces gracias al conocimiento y a la experiencia de quien diseña en el ámbito de la ingeniería, con objeto de determinar el camino a seguir para dar con la solución. Por el contrario, las salidas del diseño creativo son una interrogante en un espacio borroso, que por lo general no tiene una única alternativa inicial para la solución que pueda representar la forma final del producto. La revisión de los modelos permite apreciar efectivamente que la localización de las acciones y tareas de diseño conceptual no son tan evidentes a la hora de abordar las tareas de la fase creativa. Se observa una gran concentración y variedad de acciones y tareas en el momento de realizar las fases de diseño conceptual. Esto provoca por lo general inexactitudes en los procedimientos que se realizan en la fase creativa y mental. Como consecuencia de este análisis comparativo se pueden concluir los siguientes resultados.

\section{RESULTADOS}

Podemos concluir que las acciones y tareas que establecen la mayoría de los modelos, respecto del proceso de diseño, permiten inferir que habitualmente se dan cuatro fases típicas que definen dicho proceso: analizar, sintetizar, evaluar y ejecutar. Se admite que en la identificación de tareas la claridad de saber quién ejecuta cada etapa y en qué momento genera una discusión en torno a la importancia de los actores en el proceso se basa por lo general en la experiencia, entrenamiento y dominio del espacio conceptual de quien lo realiza o de quien ha acumulado experiencia previa.

La función y dominio del diseño industrial no están claros en el proceso, como tampoco los alcances y dominios del diseño en la ingeniería. Los modelos sugieren una clara naturaleza de realización guiada por la ingeniería a la hora de definir asociaciones en la fase conceptual y en la generación del concepto. Por otro lado, en el proceso de diseño conceptual las asociaciones que se definen en la etapa proyectual son de distinta naturaleza, como muestra la Figura 11. Están las denominadas acciones y rutinas secuenciales, que se asocian generalmente a procesos técnico-funcionales y se identifican con la ingeniería, dando resultados diferenciados desde la forma y la función; por otra parte, en el diseño creativo las acciones son de realización cíclica, con trayectorias mentales divergentes. Ambos procesos mentales tienen un fin común, pero el grado de 
Tabla 2. Modelos contemplados en la revisión. Elaboración propia.

\begin{tabular}{|c|c|c|c|c|}
\hline \multicolumn{2}{|c|}{ Modelos } & \multicolumn{2}{|l|}{ Descripción de Fases } & \multirow[b]{2}{*}{ - Producción } \\
\hline M1 & Asimow (1962) & $\begin{array}{l}\text { - Necesidades } \\
\text { - Factibilidad }\end{array}$ & $\begin{array}{l}\text { - Diseño preliminar } \\
\text { - Diseño detallado }\end{array}$ & \\
\hline M2 & Hubka-Eder (1982) & $\begin{array}{l}\text { - Diseño conceptual } \\
\text { - Orden del diseño }\end{array}$ & - Diseño de detalles & \\
\hline M3 & French (1985) & $\begin{array}{l}\text { - Necesidades } \\
\text { - Análisis del problema }\end{array}$ & $\begin{array}{l}\text { - Diseño conceptual } \\
\text { - Esquemas del diseño }\end{array}$ & - Diseño de detalles \\
\hline M4 & Gómez-Senent (1989) & $\begin{array}{l}\text { - Información } \\
\text { - Necesidades }\end{array}$ & $\begin{array}{l}\text { - Estudio preliminar } \\
\text { - Diseño básico }\end{array}$ & $\begin{array}{l}\text { - Diseño detallado } \\
\text { - Desarrollo }\end{array}$ \\
\hline M5 & Pugh (1990) & $\begin{array}{l}\text { - Mercado } \\
\text { - Especificaciones }\end{array}$ & $\begin{array}{l}\text { - Concepto de diseño } \\
\text { - Diseño de detalles }\end{array}$ & $\begin{array}{l}\text { - Fabricación } \\
\text { - Comercialización }\end{array}$ \\
\hline M6 & Gero (1990) & $\begin{array}{l}\text { - Formulación } \\
\text { - Síntesis }\end{array}$ & $\begin{array}{l}\text { - Análisis } \\
\text { - Evaluación }\end{array}$ & $\begin{array}{l}\text { - Reformulación } \\
\text { - Descripción del } \\
\text { diseño }\end{array}$ \\
\hline M7 & Cross (1994) & $\begin{array}{l}\text { - Exploración } \\
\text { - Generación }\end{array}$ & $\begin{array}{l}\text { - Evaluación } \\
\text { - Comunicación }\end{array}$ & \\
\hline M8 & Ulrich (1995) & $\begin{array}{l}\text { - Planeación del producto } \\
\text { - Desarrollo del concepto }\end{array}$ & $\begin{array}{l}\text { - Diseño del sistema } \\
\text { - Diseño de detalles }\end{array}$ & $\begin{array}{l}\text { - Pruebas } \\
\text { - Producción }\end{array}$ \\
\hline M9 & Pahl y Beitz (1996) & $\begin{array}{l}\text { - Tarea } \\
\text { - Clarificación de la tarea }\end{array}$ & $\begin{array}{l}\text { - Diseño conceptual } \\
\text { - Materialización del } \\
\text { diseño }\end{array}$ & - Diseño de detalles \\
\hline M10 & Hernandis (2003) & $\begin{array}{l}\text { - Diseño estratégico } \\
\text { - Diseño conceptual }\end{array}$ & $\begin{array}{l}\text { - Diseño de detalles } \\
\text { - Simulación }\end{array}$ & - Validación \\
\hline M11 & $\operatorname{Dim}(2006)$ & $\begin{array}{l}\text { - Definición } \\
\text { - Necesidades } \\
\text { - Diseño conceptual }\end{array}$ & $\begin{array}{l}\text { - Diseño preliminar } \\
\text { - Diseño detallado }\end{array}$ & $\begin{array}{l}\text { - Comunicación } \\
\text { - Fabricación }\end{array}$ \\
\hline
\end{tabular}

\section{PROCESO DE DISEÑO}

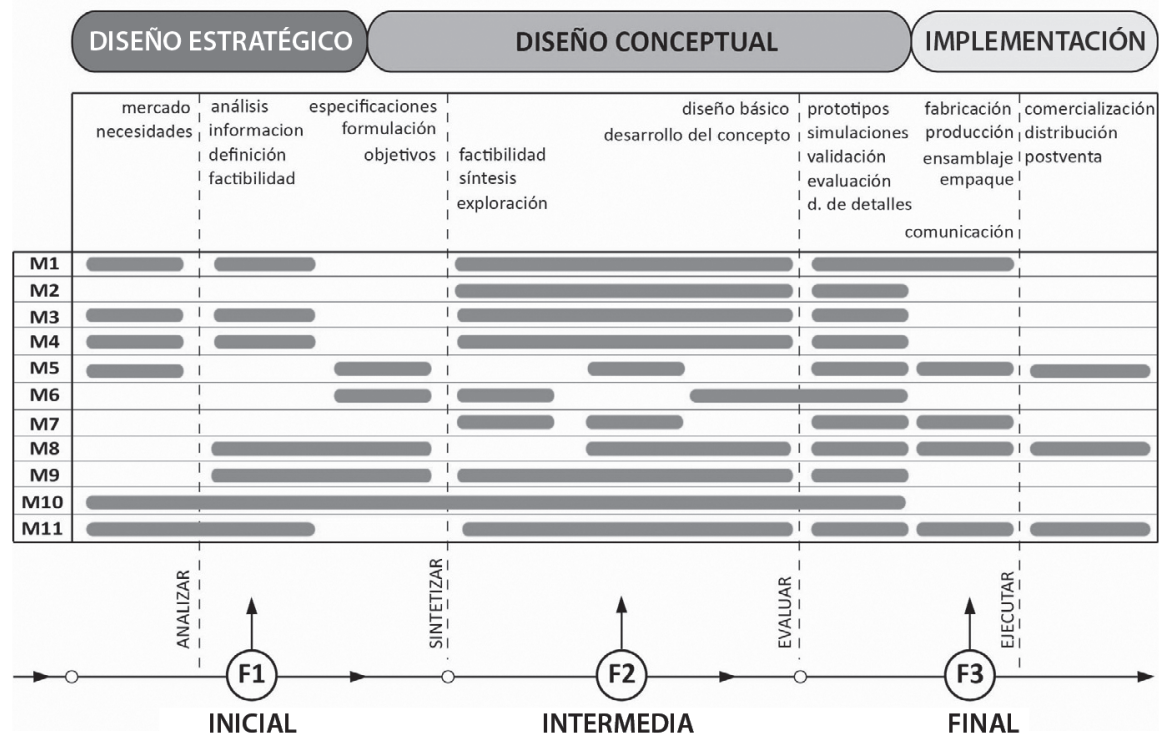

Figura 10. Comparativa de los modelos del proceso de diseño. Elaboración propia.

coincidencia no es normalmente el esperado, ya que requieren de una integración permanente durante el proceso al momento de definir el resultado del diseño. En los modelos utilizados actualmente las similitudes no están claramente definidas, así como tampoco queda claro si existe algún tipo de protocolo o formalización de procedimientos comunes que se puedan establecer y llevar a cabo 
simultáneamente a la hora de realizar las tareas y acciones del proceso de diseño.

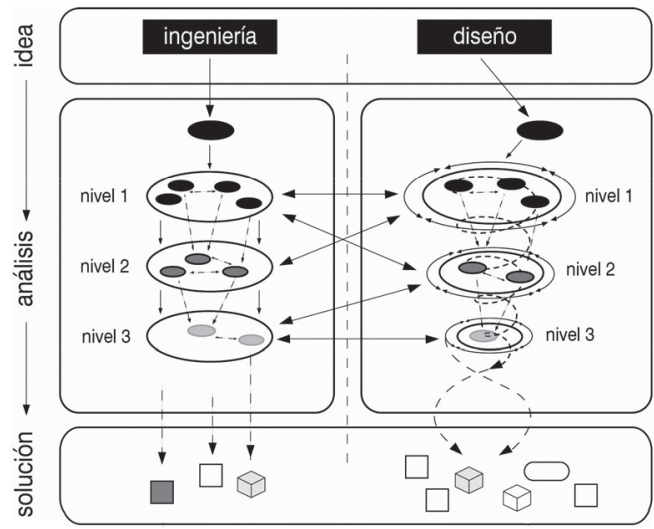

Figura 11. Pensamiento creativo en la ingeniería y el diseño. Elaboración propia.

Las estrategias de diseño permiten organizar las tareas y acciones colaborativas que se repiten permanentemente durante el proceso. Una de las dimensiones más importantes a considerar es el espacio de diseño, el cual permite representar en el dominio del objeto los aspectos y rasgos formales del concepto de diseño, como se muestra en la Figura 12, donde el dominio morfológico y el dominio funcional se encuentran al mismo nivel, lo que permite determinar el diseño de manera conjunta.

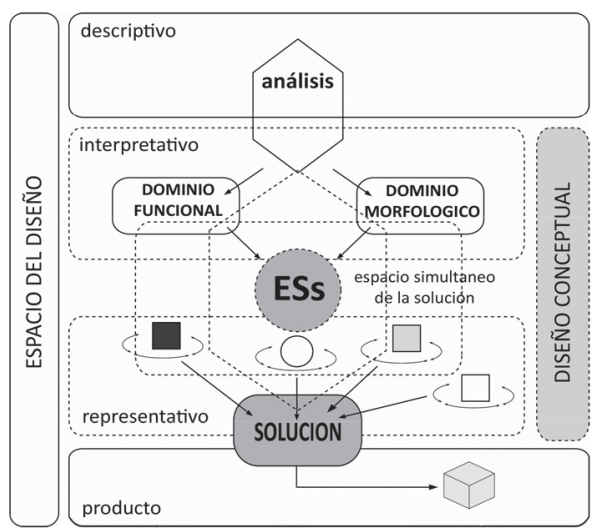

Figura 12. Modelo teórico del espacio de diseño. Elaboración propia.

Los modelos enfocados principalmente a la ingeniería suelen carecer de procedimientos orientados al proceso creativo. El diseño conceptual en la ingeniería está considerado en las fases iniciales del proceso, identificándose sobre todo con el dominio técnico y las estructuras funcionales. La solución de diseño se determina basándose en los parámetros y especificaciones iniciales, y por lo general se define el concepto de diseño en las fases preliminares del proceso de ingeniería. En la Figura 13 se aprecia que en la trayectoria del proceso de diseño las fases de entrada y salida del diseño (F1 y F3) son fases de menor duración y con poca caracterización del diseño conceptual, mientras que las fases de realización y caracterización del producto requieren de la mayor parte del tiempo para su definición (F2). Se aprecia que las tareas iniciales y finales están sistematizadas, a diferencia de aquellas pertenecientes a las fases intermedias en las que domina lo abstracto y lo conceptual.

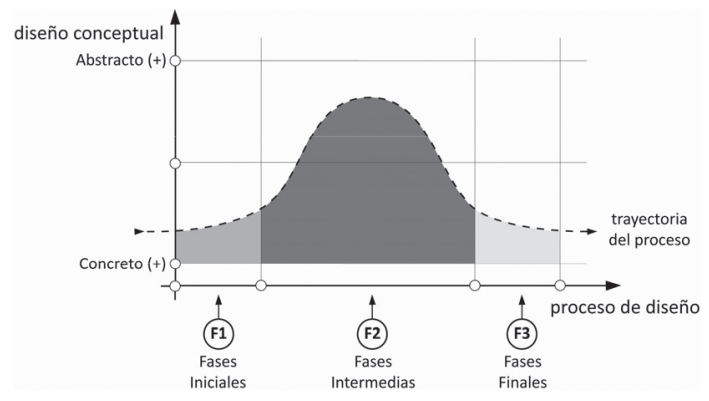

Figura 13. Trayectoria del proceso de diseño. Elaboración propia.

Aunar criterios y organizar la información en la entrada de datos del proceso de diseño, así como la implementación de estos en la fase conceptual, son objetivos fundamentales a lo hora de diseñar. Los modelos a utilizar deben relacionar los factores significativos de la expresión formal para establecer las transiciones necesarias con objeto de optimizar el proceso de diseño en el propio espacio conceptual.

\section{CONCLUSIONES}

La experiencia se considera como la principal característica a la hora de diseñar y se asume como una garantía a la hora de aplicar los procedimientos y acciones necesarias durante el proceso de diseño. Por otro lado la falta de integración y de coherencia en el uso de un lenguaje común es un factor determinante si se pretende establecer un diálogo multidisciplinario. Esta debilidad, que siempre está presente, es independiente de los métodos que se utilicen en el proceso de diseño. Aparentemente pareciera 
ser que quien lo tiene más claro es la ingeniería, ya que responde a acciones resolutivas desde los sistemas técnicos y mecánicos. El logro de los objetivos propuestos suele ser bajo esta orientación, la que es más rigurosa y comprobable, teniendo en cuenta que la generación de documentación técnica de respaldo permite su validación en cualquier momento del proceso. Por lo general la búsqueda se centra principalmente en los buenos resultados de diseño que se dieron en el pasado, volviendo a aplicarlos en las nuevas soluciones.

Las acciones y trayectorias concepto-forma, en el proceso de diseño, son una práctica a veces especulativa y se proyectan generalmente en un contexto del diseño referido a la satisfacción del usuario/consumidor y al significado simbólico del producto. La incorporación de nuevas variables sensoriales y perceptivas como la emoción, las sensaciones, la experiencia, y las relaciones con criterios de personalización, son cada vez más recurrentes y necesarias hoy cuando se diseña un producto. La originalidad de los diseños también depende de la información que se genera en el proceso; las ideas y representaciones más novedosas dan lugar a diseños más originales, pero generalmente las soluciones propuestas son difusas y muchas veces inexplicables.

El diseñador debe tener la capacidad de generar soluciones centradas fundamentalmente en el usuario/consumidor, con un marcado carácter social, cultural y de identidad con los objetos cotidianos. En este aspecto la búsqueda se realiza con carácter exploratorio y experimental. Enriquecer y compartir las nuevas experiencias con los paradigmas actuales del proceso de diseño es deseable, y genera nuevas expectativas en torno a aquellas experiencias asociadas al diseño conceptual y al proceso creativo. Aminorar el grado de incertidumbre para dar forma al concepto, compartir los espacios de búsqueda y de inspiración en la ingeniería y diseño conceptual es uno de los objetivos que resolvería al mismo tiempo problemas de comunicación y registro de información para una buena gestión del conocimiento futuro.

La integración de los procesos de ingeniería con los procesos de diseño creativo deben ser capaces de evolucionar hacia modelos más eficientes que aúnen criterios, contemplando los paradigmas actuales relacionados con el diseño y rediseño de productos. Los modelos tradicionales utilizados convencionalmente en el proceso de diseño muestran un alto grado de obsolescencia, quedándose atrás respecto del dinamismo del mercado y de la participación activa del consumidor, especialmente considerando lo referente a la toma de decisiones estratégicas de las empresas, ya que es cada día más evidente la influencia de las buenas decisiones de estas en torno al éxito o fracaso de nuevos diseños y productos, donde participa el usuario.

El diseño exige actualizarse, los modelos deben ser capaces de mutar hacia las nuevas expectativas, experiencias y solicitudes de las empresas, usuarios y consumidores. Los modelos actuales, con los que se aborda y desarrolla el proceso de diseño, son los mismos que se han utilizado por mucho tiempo y deben ser revisados, así como también los marcos referenciales considerados hasta ahora. También se requiere contar con métodos, técnicas y herramientas que permitan resolver interdisciplinariamente, desde la ingeniería y el diseño, de una manera más rápida e integral las propuestas y definición del diseño. Para que esto ocurra se requiere de modelos multidisciplinarios que otorguen la posibilidad de hacerlo, y que faciliten, en la medida de lo posible, la realización integrada del proceso de diseño.

\section{REFERENCIAS}

[1] T.J. Howard, S.J. Culley and E. Deconinck. "Describing the Creative Design Process by the Integration of Engineering Design and Cognitive Psychology Literature". Design Studies. Vol. 29, pp. 160-180. 2008.

[2] O. Rivka. "Educating the Designerly Thinker". Design Studies. Vol. 20, Issue 2, pp. 113-114. 1999.

[3] N. Cross. "Métodos de Diseño: estrategias para el diseño de productos". Ed. Limusa, pp. 11-31. 2002.

[4] M.J.French. "Conceptual design for Engineers". The Design Council, pp. 1-5. 1999.

[5] S. Pugh. "Total Design". Ed. Prentice Hall, pp. 3-7. 1991.

[6] P. Suh Nam. "Designing in of Quality Trough Axiomatic Design". IEEE Transactions of Reability. Vol. 44, Issue 2, pp. 256-257. 1995.

[7] C. Dym y P. Little. "El Proceso de Diseño en Ingeniería". Editorial Limusa-Wiley, pp. 30-41. 2006. 
[8] J.C. Briede. "La Metodología Sistémica y el rol del Boceto en el Diseño Conceptual de Productos Industriales". Tesis para optar al grado de doctor. Universidad Politécnica de Valencia, p. 35. Valencia, España. 2005.

[9] R. León Moran. "Sistémica aplicada al Diseño de Productos en Venezuela". Tesis para optar al grado de doctor. Universidad Politécnica de Valencia, p. 29. Valencia, España. 2009.

[10] M. Tobey. "Styling and Design: Intuition and Analysis in Industrial Design". Design Studies. Vol 18, Issue 1, pp. 8-9. 1997.

[11] K. Ulrich y S.D. Eppinger. "Diseño y Desarrollo de Productos: enfoque multidisciplinario". $3^{\mathrm{a}}$ Edición. Ed. McGraw-Hill. México. 2004.

[12] E. Mulet. "Análisis Experimental y Modelización Descriptiva del Proceso de Diseño". Tesis para optar al grado de doctora. Universidad Politécnica de Valencia, pp. 4-5. Valencia, España. 2003.

[13] K. Otto and K Wood. "Product Design, Techniques in Reverse Engineering and New Product Development". Editorial Prentice Hall, pp. 148-153, 414-419. 2001.

[14] B.J. Chaur. "Diseño Conceptual Asistido por Ordenador: Un estudio analítico sobre aplicaciones y definiciones de la Estructura básica de un Nuevo programa". Tesis para optar al grado de doctor. Universidad Politécnica de Cataluña, p. 36. Barcelona, España. 2004.

[15] C. Dym and A. Agogino. "Engineering Design Thinking, Teaching and Learning". Journal of Engineering Education, p. 104. 2005.

[16] G. Pahl and W Beitz. "Engineering Design a Systematic Approach". Ed. Springer Verlag, p. 86. 1996.

[17] D. Ullman. "The Mechanical Design Process". Ed. McGraw-Hill International. New York, Unites States. 1997.

[18] J. Gero. "Computational Models of Innovative and Creative Design Process". Technological
Forecasting and Social Change. Vol. 64, pp. 183-196. 2000.

[19] B. Hernandis and J.C. Briede. "An Educational application for a product design and engineering systems using integrated conceptual models". Ingeniare. Revista chilena de ingenieria. Vol. $17 \mathrm{~N}^{\circ}$ 3, pp. 432-442. 2009.

[20] A. Wodehouse and J. Ion. "Information use in Conceptual Design: existing taxonomies and new approaches". International Journal of Design. Vol. 4, Issue 3, pp. 53-65. 2010.

[21] A. Hevner and S. Chatterjee. "Design Research in Information Systems". Integrated Series 145 in Information Systems. Vol. 22, p. 145. Springer Science- Business Media. 2010.

[22] E. De Bono. "El pensamiento creativo". Ediciones Paidós, pp. 13-44, 112-113. España. 2007.

[23] A. Esteve de Quezada. "Creación y Proyecto". Ed. Institución Alfonso el Magnánimo, p. 31. Valencia, España. 2001.

[24] H.P. Casakin. "Factors of metaphors in design problem-solving: Implications for design creativity". International Journal of Design. Vol. 1, Issue 2, p. 21. 2007.

[25] C. Eckert and M. Stacey. "Sources of Inspiration: a language of design". Design Studies. Vol. 21, pp. 523-538. 2000.

[26] E. Gómez-Senent. "La ciencia de la Creación de lo Artificial". Ed. SPUPV. Universidad Politécnica de Valencia, p. 51. España. 1998.

[27] E. Mancini. "La materia de la Invención, Materiales y Proyectos". Ed. CEAC, pp. 47-60. Barcelona, España. 2000.

[28] P. Plsek. "Working Papers: Models for the Creative process". Date of visit: July 9, 2013. URL: http://www.directedcreativity. com/pages/WPModels.html

[29] M. Asimow. "Introduction to Design". First Edition. Ed. Prentice Hall, p. 12. 1962.

[30] A. Hatchuel and B Weil. "C-K Design Theory: and advanced formulation". Springer-Verlag, London Limited. Vol. 19, pp. 181-186. 2009. 